\title{
BREVE RESEÑA HISTÓRICA DE LAS VÍAS EN COLOMBiA
}

\author{
Ismael Osorio Baquero ${ }^{1}$
}

${ }^{1}$ Docente, Universidad Cooperativa de Colombia, Villavicencio, Colombia

Correo electrónico: isosba@yahoo.es

Recibido: 30 de septiembre del 2013. Aprobado: 18 de enero del 2014.

Cómo citar este artículo: I. Osorio, "Reseña histórica de las vías en Colombia". Ingeniería Solidaria, vol. 10, n. 17, pp. 183-187, en.-dic., 2014. doi: http://dx. doi. org/10.16925/in.v10i17.880

Resumen. El indígena precolombino desarrolló en grado variable la capacidad de desafiar y ejecutar obras en el ramo vial. Para el caso que concierne, se señalan como las más importantes, la construcción de distintas clases de puentes y la de embarcaciones. Asimismo, los caminos que van en línea recta cualquiera que sea la pendiente, se explican por la necesidad de llegar cuanto antes a un punto determinado, pues siendo gente de a pie y ágil, la pendiente no representaba obstáculo. Antes, hubo caminos... Anduvieron los precolombinos comerciando intercambiando "trueque" más que imponiendo una lengua común. Por haber desarrollado sus civilizaciones sobre las cordilleras, su arte de construir trochas y puentes, de llegar a mercados distantes escalando montes y cruzando ríos los llevó a hacer puentes de bejucos, y a empedrar caminos y hacer escaleras tan perfectas como las de los taironas, y a cruzar provincias de lenguas diversas como en los largos caminos reales que recorrían los chasquis incas yendo de tambo en tambo a velocidad de mulas, como ocurría también entre los aztecas.

Palabras clave: caminos precolombinos, carreteras nacionales, vías férreas, Colombia.

\section{Short Historical Summary of Roads in Colombia}

Abstract. Pre-Columbian indigenous peoples developed a variable degree of capacity to undertake and execute road works. Their most important abilities involved building diverse types of bridges and boats. Paths that run in a straight line, no matter the incline, stem from the need to arrive at a certain place as soon as possible; because they were physicallyfit walkers, inclines were no obstacle. In the past, there were paths. The pre-Columbians traveled over them to trade and "barter" more than in an attempt to impose a shared language. Because they had developed their civilizations in the mountain ranges, their artistry in building trails and bridges, reaching distant markets by climbing mountains and crossing rivers led them to create bridges using vines, to build stone pathways and stairs as perfect as those of the Taironas, and to cross provinces with diverse languages such as the royal pathways used by the Inca chasquis (messengers) when traveling between farms at the speed of mules, which also happened among the Aztecs.

Keywords: pre-Columbian paths, national roads, railways, Colombia.

\section{RESENHA histórica bReVe dAS VIAS NA Colômbia}

Resumo. O indígena pré-colombiano desenvolveu em grau variável a capacidade de desafiar e executar obras na rede viária. Para o caso que concerne, indicam-se como as mais importantes a construção de diferentes tipos de pontes e a de embarcações. Além disso, os caminhos que vão em linha reta, qualquer que seja a inclinação, o que não representava um obstáculo devido à agilidade dessas pessoas, explicam-se pela necessidade de chegar o quanto antes a um ponto determinado. Caminhando, os pré-colombianos comercializaram, intercambiaram mais que imporem uma língua comum. Por terem desenvolvido suas civilizações sobre as cordilheiras, sua arte de construir estradas e pontes, de chegar a mercados distantes escalando montanhas e cruzando rios os levou a fazerem pontes de cipós, a empedrar caminhos e fazer escadas tão perfeitas quanto as dos taironas, e a cruzar províncias de línguas diversas como nos longos caminhos reais que percorriam os chasquis incas indo de estábulo em estábulo à velocidade de mulas, como ocorria também entre os astecas.

Palavras-chave: caminhos pré-colombianos, estradas nacionais, vias férreas, Colombia. 


\section{Introducción 1}

Grandes tramos de las carreteras actuales de Colombia se trazaron siguiendo los caminos reales coloniales, de igual manera que estos mejoraron las condiciones de trochas y caminos indígenas. Buena parte de esta superposición de rutas obedece a las características de nuestra topografía, donde se alternan valles, ríos y cordilleras que obligan a buscar los puntos más accesibles para salvar las alturas y los puntos de mayor estrechez en el cauce de los ríos, para vadearlos o construir los puentes.

La determinación del período cobijado por la legislación se definió por la primera ley que expidió el nuevo gobierno republicano de Colombia y la creación del Ministerio de Obras Públicas en 1905, durante el gobierno del general Rafael Reyes que paulatinamente entraría a modernizar, haciendo más operativa y eficiente la construcción de caminos, carreteras, ferrocarriles y edificios. A diferencia de nuestros antiguos caminos de herradura.

\section{Caminos de herradura (Colonia)}

Un camino real español se diferencia del camino de los indios en que van a transitarlo, además de los hombres, los caballos, los bueyes, las mulas y los indios cargueros (figuras 1 y 2). Con los españoles va a llegar también la rueda, pero el camino real no va a ser carreteable sino mucho tiempo después, en el altiplano y ya en la república. Será un camino real republicano. Los caminos

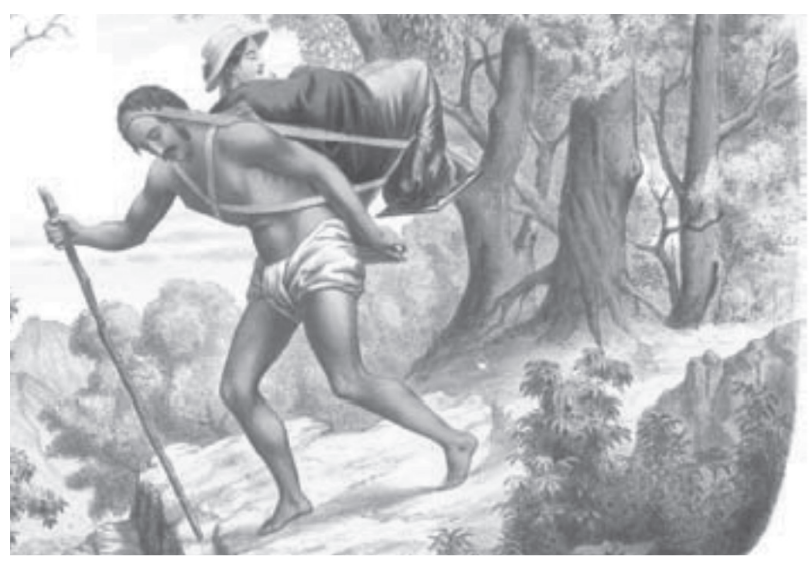

Figura 1. Carguero indígena

Fuente: [2]

1 Este artículo se deriva de una ponencia presentada en el I Congreso Nacional de Vías, realizado en noviembre de 2013, en Medellín (Antioquia), Colombia.

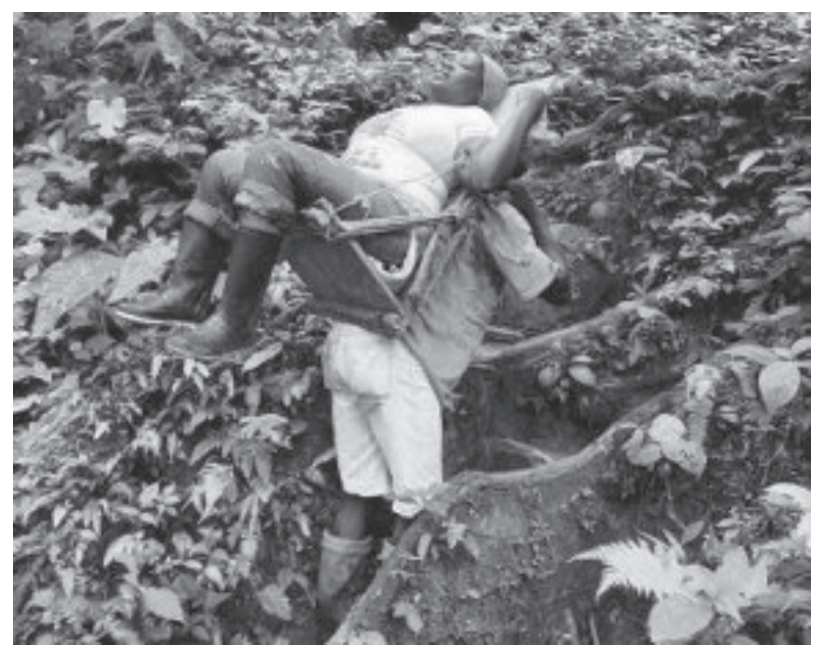

Figura 2. Pasero de la actualidad (Baudó-Choco)

Fuente: [3]

reales más importantes serán de herradura y llegar a un puente de arcos romanos como el Puente del Común sobre el Bogotá, es cosa ya para los virreyes que llegan tarde. Los primeros caminos reales se parecen más a los de los incas, lo mismo que los primeros puentes [1].

\section{Caminos reales (Nueva Granada)}

Dentro de la red de caminos coloniales, los más importantes eran los llamados caminos reales, ellos, además de ser los de mayor circulación, unían las diferentes provincias y su mantenimiento y administración se realizaba por delegación real (figura 3).

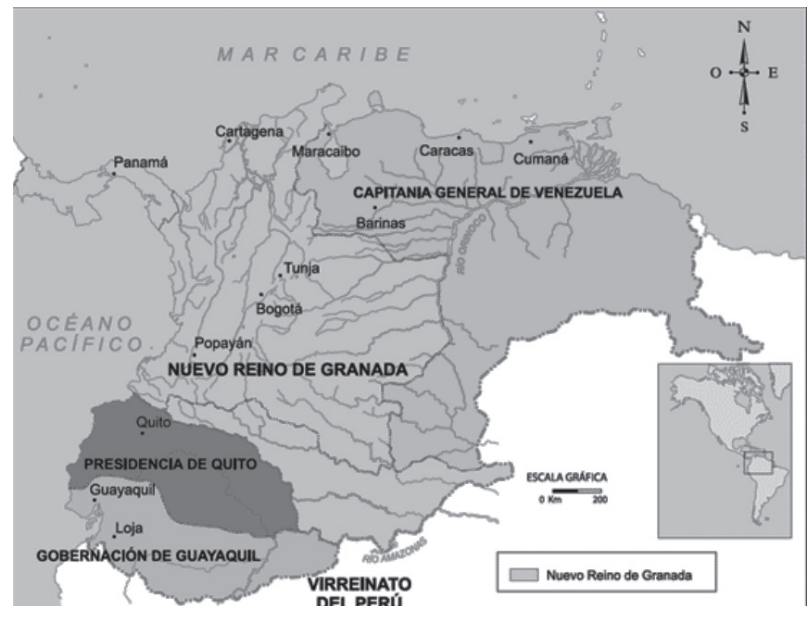

Figura 3. La Nueva Granada

Fuente: [4] 
En términos de las condiciones materiales, los caminos reales eran concebidos como caminos de herradura, en tanto que los demás eran generalmente caminos de a pie, lo que no obsta para señalar que buena parte de los caminos reales fueron frecuentemente intransitables para bestias de silla o carga, debiendo utilizarse los indígenas en tales menesteres.

El primer camino para llegar a Cundinamarca procedente de la costa Atlántica fue el Opón, por donde también empieza su historia hispano-india y entró el primer destello de la civilización de Occidente, pues en aquellas épocas antiguas las guerras eran portadoras de civilización y de cultura.

\section{Carreteables (República de Colombia)}

Hacia 1846 empezó en Bogotá el transporte urbano de mercancías, muebles, entre otros, en carros tirados por bueyes y caballos, y fue objeto de protestas hasta 1859, por daños en edificios y cañerías. En el año de 1856, había hasta doscientos. En 1869, destrozaban las calles. La escasez de vehículos se atribuyó, en parte, a que los atanores que conducían las aguas eran de barro, por falta de los de hierro, y por eso no soportaban cargas pesadas. En el siglo XIX, entre Bogotá y Facatativá circulaban al año más de 32000 carros, con cargas de una tonelada en promedio. Hacia 1887 había en la Sabana unos 2000 carros de bueyes. La Ley del 28 de mayo de 1864, que puede considerarse como el primer paso fundamental en la estructuración de un plan vial integral, fue completada con la Ley 52 de 1872, en la época de la segunda presidencia de Murillo Toro. En 1873 hubo una verdadera fiebre vial en toda Colombia. Se considera como carreteable una vía afirmada de menos de 4,6 metros de banca, y como carretera, la que sobrepase esta anchura [6].

Una de las primeras que se construyeron en Colombia fue la de Cúcuta a Los Cachos, sobre el río $\mathrm{Zu}$ lia, entre 1865 y 1875; desde este último año, a raíz del terremoto que asoló a la ciudad, entró al servicio a pesar de no estar terminada. El Carreteable de Cambao iniciado por cuenta del Estado de Cundinamarca en 1882, pasó a la Nación en 1886 con motivo de la Reforma. Tiene 96 kms., con un ancho de 3 metros, después hubo que ensancharlo para permitir la introducción de los elementos del Ferrocarril de la Sabana. El camino por el que se iba de Santafé a Quito, pasando por Cartago, tenía una longitud en el siglo XVI de 221 le- guas que, discriminada por las distancias entre las ciudades principales. En este trayecto, el camino tenía tres derivaciones de importancia: el camino Cali - Buenaventura, el camino Pasto - Barbacoas - Tumaco, y el camino Cartago - Chocó [7].

Partiendo de Santafé hacia la Gobernación de Popayán y la Presidencia de Quito, se tomaba rumbo a Mariquita e Ibagué por una de dos rutas: una que se dirigía hacia el Magdalena, pasando por Villeta, Honda, Guaduas y Mariquita; y otra que por Funza, Tena, La Mesa y Tocaima, iba hacia Ibagué. En esta segunda ruta, y desde Tocaima, fue durante mucho tiempo indispensable pasar por Honda y Mariquita para llegar a Ibagué.

\section{Las vías en Colombia}

\subsection{Vías férreas}

La primera vía férrea que se construyó en Colombia fue la de Panamá, sería la primera de índole interoceánica al enlazar los océanos Pacífico y Atlántico; debería superar la divisoria de aguas entre ambos mares. La vía del ferrocarril de Panamá, de 77 kilómetros de largo, se concluyó en 1855, construida inicialmente en trocha de $1435 \mathrm{~m}$, la que ahora se llama normal, pero casi de inmediato fue pasada a cinco pies, 1524 metros. Colombia perdió ese su primer ferrocarril en 1903, con la separación de Panamá. En 1855 don Ramón Santodomingo Vila y don Raúl Jimeno recibieron del Estado de Bolívar la concesión para construir un ferrocarril de Barranquilla hasta Sabanilla, ya a la orilla del mar. Luego del traspaso de la concesión, una empresa alemana inició la construcción y llegó a la actual Colombia la primera locomotora, con el nombre de "Bolívar".

El 22 de septiembre de 1908 llegó el Ferrocarril de Girardot a Facatitavá y se realizó la primera comunicación férrea de la Capital de la República con el Magdalena, con el tipo de maquinaria que se muestra en la figura 4, el de Girardot fue concluido en trocha de yarda. Al año siguiente, con ayuda de locomotoras apropiadas a esa vía, una de las más difíciles del mundo, no sólo de la continuación del Ferrocarril del Cauca, ya denominado del Pacífico. En 1911 es ya el departamento de Caldas, que inició gestiones para comunicar a Manizales con el río Cauca [12]. 


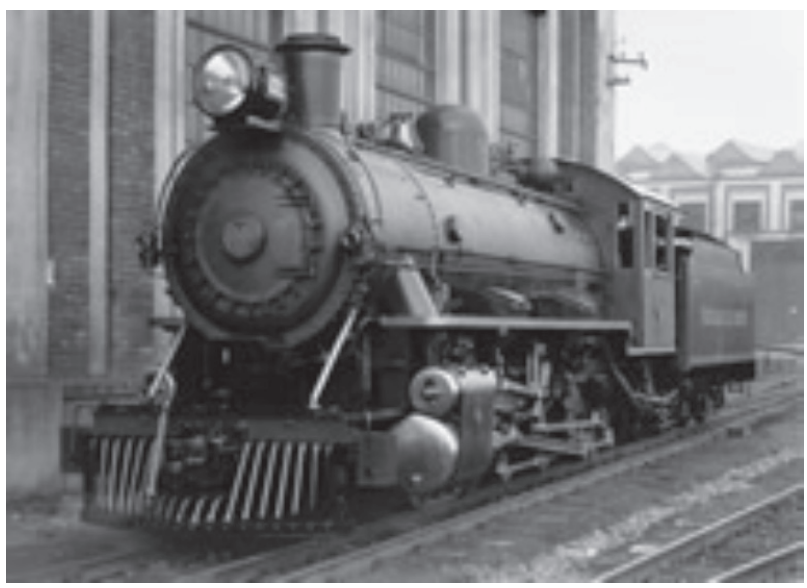

Figura 4. Ferrocarril del Pacífico Fuente: [8]

\subsection{Longitud aproximada de las carreteras en Colombia (1994)}

En el año 1997, Colombia poseía aproximadamente 5700 kilómetros en carreteras troncales (tabla 1), 4100 kilómetros de carreteras transversales (tabla 2), para un total de 11287 kilómetros pavimentados y 5076 sin pavimentar (tabla 3).

Tabla 1. Rutas troncales nacionales

\begin{tabular}{|l|c|}
\hline \multicolumn{1}{|c|}{ Troncales } & Longitud (Km) \\
\hline De Occidente & 1778 \\
\hline De Magdalena & 1597 \\
\hline Central & 476 \\
\hline De Urabá & 358 \\
\hline Del Eje cafetero & 215 \\
\hline Villavicencio - Saravena & 1258 \\
\hline
\end{tabular}

Fuente: [9]

Tabla 2. Rutas transversales nacionales

\begin{tabular}{|l|c|}
\hline \multicolumn{1}{|c|}{ Transversales } & Longitud (Km) \\
\hline Del Caribe & 891 \\
\hline Carmen de Bolívar - Valledupar - Maicao & 441 \\
\hline Medellín - Cúcuta - Pto. Santander & 649 \\
\hline Medellín - Bogotá & 566 \\
\hline Buenaventura - Villavicencio & 724 \\
\hline Huila - Cauca & 199 \\
\hline Tumaco - Mocoa & 413 \\
\hline La Mina - Río Caguán & 189 \\
\hline
\end{tabular}

Fuente: [9]
Tabla 3. Inventario de la red vial nacional

\begin{tabular}{|c|c|c|}
\hline \multirow{2}{*}{ Nombre de la regional } & \multicolumn{2}{|c|}{ Longitud en Km. } \\
\hline & Pavimentados & Sin pavimentar \\
\hline Antioquia & 1439 & 121 \\
\hline Atlántico & 272 & 0 \\
\hline Bolívar & 471 & 0 \\
\hline Boyacá & 525 & 492 \\
\hline Caldas & 302 & 0 \\
\hline Caquetá & 288 & 97 \\
\hline Casanare & 578 & 191 \\
\hline Cauca & 423 & 868 \\
\hline Cesar & 549 & 180 \\
\hline Chocó & 23 & 280 \\
\hline Córdoba & 487 & 95 \\
\hline Cundinamarca & 620 & 141 \\
\hline Guajira & 277 & 49 \\
\hline Huila & 429 & 422 \\
\hline Magdalena & 350 & 314 \\
\hline Meta & 431 & 480 \\
\hline Nariño & 558 & 222 \\
\hline Norte de Santander & 432 & 173 \\
\hline Putumayo & 11 & 353 \\
\hline Quindío & 164 & 0 \\
\hline Risaralda & 207 & 85 \\
\hline Santander & 859 & 396 \\
\hline Sucre & 188 & 89 \\
\hline Tolima & 548 & 0 \\
\hline Valle & 627 & 0 \\
\hline Ocaña & 229 & 28 \\
\hline Total de kilómetros & 11287 & 5076 \\
\hline
\end{tabular}

Fuente: [9]

\section{Conclusiones}

- La colonización del territorio colombiano por los españoles se desarrolló a través de la vía natural a lo largo del río Magdalena y el río Cauca. Desde entonces, la historia reseña lo difícil que es movilizarse por el país, a través de las vías construidas siguiendo los caminos de la colonia.

- Desde ese entonces las actividades políticas, económicas y culturales del país se concentraron principalmente en la zona Andina, conformada por tres cordilleras, cuyas laderas y valles sirven de cauce a los ríos Cauca y Magdalena. Otros centros de tales actividades son los litorales Atlántico y Pacífico. 
- Colombia es uno de los países en Latinoamérica más atrasados en materia de infraestructura de transporte, según el informe presentado por Fedesarrollo en el marco de la novena versión del Congreso Nacional de Infraestructura.

\section{Agradecimientos}

Mis más sinceros agradecimientos a la Universidad Cooperativa de Colombia-UCC, al comité organizador del Primer Congreso Nacional de Vías, a las directivas de la UCC-sede Villavicencio, decana (E) Dra. María Lucrecia Ramírez Suárez; por la oportunidad de participar en tan importante evento.

\section{Referencias}

[1] G.Arciniegas, “Caminos Reales deColombia”. [En línea]. Disponible en: http://es.scribd.com/doc/62742856/Caminos-Reales-de-Colombia

[2] Fundación Estanislao Merchán-Cano. Isidoro Medina Patiño. [En línea]. Disponible en: http://www.isidorohistoria.com/historia/

[3] F. Pinzón, “Galería de fotos”. Periódico El Tiempo. [En línea]. Disponible en: http://www.eltiempo.com/Multimedia/galeria_fotos/carrusel/el-rollo-de-fili-atlaschocoano_9565805-5

[4] La historia con mapas, "Gran Colombia 1819-1830: el sueño de Bolívar”. [En línea]. Disponible en: http:// www.lahistoriaconmapas.com/america/colombia/ gran-colombia/
[5] R. Velandía, "La trocha del Opón”, en Caminos reales de Colombia, M. Useche, Ed. [En línea]. Disponible en: http://admin.banrepcultural.org/node/71231

[6] V. M. Patiño, Historia de la cultura material en la América equinoccial: vías, transportes, comunicaciones, vol. 3. Bogotá: Instituto Caro y Cuervo, 1990-93. [En línea]. Disponible en: http://www.banrepcultural.org/blaavirtual/historia/equinoccial_3_transportes/indice.htm

[7] R. D. Londoño, "Aportes y perspectivas de la ingeniería en el desarrollo humano en Colombia, en el devenir de los últimos 200 años". Revista Épsilon, n. ${ }^{\circ} 16$, pp. 11-30, enero-junio 2011.

[8] J. Arias de Greiff, "Ferrocarriles en Colombia 18361930”. Credencial Historia, n. ${ }^{\circ}$ 257. [En línea]. Disponible en: http://admin.banrepcultural.org/blaavirtual/ revistas/credencial/mayo2011/ferrocarriles-en-colombia-1836-1930

[9] W. Muñoz, "Concesiones viales en Colombia: historia y desarrollo”. Tecnura 10, 2002. [En línea]. Disponible en: http://tecnura.udistrital.edu.co/downloads/revista10/ pdf/imagenes/concesiones_viales.pdf 